\title{
Michèle Virol dir., Les Oisivetés de Monsieur de
} Vauban

édition intégrale, Seyssel, Champ Vallon, 2007, 1722 pages.

Hélène Vérin

\section{OpenEdition \\ Journals}

Édition électronique

URL : http://journals.openedition.org/dht/1102

DOI : 10.4000/dht.1102

ISSN : $1775-4194$

Éditeur :

Centre d'histoire des techniques et de l'environnement du Cnam (CDHTE-Cnam), Société des élèves du CDHTE-Cnam

\section{Édition imprimée}

Date de publication : 1 juin 2008

Pagination : $217-219$

ISBN : 978-2-95-30779-1-9

ISSN : 0417-8726

Référence électronique

Hélène Vérin, " Michèle Virol dir., Les Oisivetés de Monsieur de Vauban », Documents pour l'histoire des techniques [En ligne], 15 | $7^{\text {er }}$ semestre 2008, mis en ligne le 22 octobre 2010, consulté le 22 septembre 2020. URL : http://journals.openedition.org/dht/1102 ; DOI : https://doi.org/10.4000/dht.1102 


\section{Michèle Virol dir., Les Oisivetés de Monsieur de Vauban}

édition intégrale, Seyssel, Champ Vallon, 2007, 1722 pages.

Hélène Vérin

\section{RÉFÉRENCE}

Michèle Virol dir., Les Oisivetés de Monsieur de Vauban, édition intégrale, Seyssel, Champ Vallon, 2007, 1722 pages.

1 L'édition des Oisivetés de Monsieur de Vauban ou ramas de plusieurs mémoires de sa façon sur différents sujets, est un événement qui mérite toute notre attention. Bien sûr, elle comble d'abord une absence, cruelle aux admirateurs du grand homme comme aux historiens, celle de l'intégralité des vingt neuf mémoires qu'il écrivit et rassembla, offerte pour la première fois, au public. Mais encore, ce volume de plus de 1700 pages, présenté dans un joli coffret, sous une forme maniable et, soulignons-le, à un prix abordable, agréable à lire et bien illustré, a d'autres vertus. Outre l'introduction du maître d'œuvre, Michèle Virol, elle propose, pour chacun des mémoires une présentation et des notes critiques établies par vingt chercheurs qui mettent leurs compétences particulières au service du lecteur du XXI ${ }^{e}$ siècle.

2 Ne boudons pas notre plaisir. Leurs éclaircissements, leur souci de mettre en perspective chacune des multiples facettes des curiosités et de l'esprit de Vauban enrichissent notre lecture et donnent à tous des clefs pour en saisir l'actuel intérêt. Ils nous aident à mesurer l'apport de Vauban à toutes sortes de réflexions, de méthodes, de sciences qui, inconnues ou s'ébauchant à peine en son temps, devaient se développer ultérieurement. Ce beau travail collectif doit être salué.

3 Il nous convie à la découverte d'un gentilhomme au service du grand roi, au service d'un royaume auquel il a donné le meilleur de lui-même, soucieux que l'on traite au plus juste la multitude de ses habitants pour lesquels il s'ingéniait à penser les conditions d'un mieux possible. Non que Vauban soit, à vrai dire, un homme des 
Lumières avant la lettre. Sur le fond, il apparaît dans ses Oisivetés, plus près de Galiani que de Diderot, sans illusion sur ses contemporains, mais aussi sans l'ombre d'un ressentiment. Homme de principe, attaché à l'ordre établi, mais soucieux que celui-ci serve le royaume et que chacun, du roi au plus obscur ouvrier occupe au plus juste, et au mieux, sa place. S'il développe l'idée d'une excellente noblesse (tome II) c'est comme moyen de la voir bien servir les intérêts du royaume et s'il calcule au plus juste le salaire d'un ouvrier terrassier (tome XI), c'est pour maîtriser les dépenses dans les chantiers du roi et ramener à une juste valeur le profit de l'entrepreneur.

Les Oisivetés de Vauban, ce sont ces écrits qu'il a composés dans le temps libre que lui laissait - on ne sait par quel miracle - des obligations incessantes et harassantes. Oisivetés donc, au sens latin de l'otium, c'est-à-dire cette part de la vie qui n'est pas consacrée aux offices, aux obligations et aux responsabilités du métier, qui permet de s'adonner à d'autres réflexions qui ne sont pas en prise directe sur les tâches à accomplir, qui ouvrent un temps à la pensée, et d'autres espaces. Car cet ingénieur ordinaire du roi (1655), devenu gouverneur de la citadelle de Lille (1668 et 1683), commissaire général des fortifications (1678), lieutenant général des armées du roi (1688) et enfin maréchal de France (1703), a abordé, dans ses Oisivetés, au fil du temps, des circonstances ou des pensées qui lui venaient à l'esprit, un champ très large de sujets qui tous, intéressent le royaume.

5 Le volume publié suit l'ordre de présentation des Mémoires voulu par Vauban. Bon nombre d'entre eux ont trait à la guerre, aux moyens de la conduire et de l'améliorer pour augmenter, stabiliser ou plus tard sauvegarder la puissance du royaume. Il s'achève sur un " projet de paix assez raisonnable » dont le titre même indique et les espoirs dont il est porteur et le doute de voir jamais, en telles affaires, triompher la raison. Il exprime des constantes dans la démarche de Vauban. Partant de la déploration du «malheureux enchaînements de guerres qui n'ont fait, depuis 40 ou 50 ans que se succéder les unes aux autres (p. 1717), se donnant pour objectif de « contenter » tous les intéressés, en sorte que chacun « n'en serait que mieux », il décrit ce que serait, en l'état présent des choses, la meilleure proportion entre puissance et équilibre des forces en Europe. Parmi ces mieux, raisonnablement évalués, le fameux «la France a des bornes naturelles au-delà desquelles il semble que le bon sens ne permette pas de porter ses pensées» (p. 1706). Vauban s'y exprime, comme à son habitude, sans complaisance. Soucieux d'allier sincérité et efficacité du raisonnement, il va jusqu'au bout de ses idées, sans « refuser à mes pensées, conclut-il, de se produire au fur et à mesure qu'elles se sont présentées » (p. 1719). Cette dernière phrase pourrait illustrer sa démarche dans l'ensemble des mémoires qu'il a menés à terme.

6 Partant de problèmes circonstanciés, il avance dans ses analyses, élargissant le champ de ses investigations pour les inclure dans des considérations plus vastes, toujours en vue d'efficacité. Ainsi, abordant des projets d'ingénieur dont il a la charge, il cherche à en calculer les effets dans l'espace et le temps, pour les habitants en général. Le projet de canal du Languedoc (tome I) l'amène à des réflexions sur le commerce. On encore, devant la pénurie de bois à bâtir pour les bâtiments civils, la fortification et la marine, il s'applique à composer un traité de la culture des forêts (tome IV) qui en propose la gestion rationnelle sur quelque deux cents ans, précisant comment en maintenir la population en réglant les coupes et en établissant des pépinières. C'est une caractéristique de sa vision de l'action que d'associer les exigences du court, du moyen et du long terme. S'il est toujours attentif à l'adéquation hic et nunc, il demeure le porte- 
parole de l'intérêt du royaume et de son inscription dans la durée. Il s'agace du « peu de vue » des particuliers, tout occupés de la jouissance immédiate de la forêt et négligeant leurs intérêts de régnicoles. Ce qui ne l'empêche pas de reconnaître leur « droit naturel " à jouir de leurs biens. Sa solution : rechercher, en s'appuyant sur des supputations méthodiquement calculées, comment dépasser cette contradiction en n'apportant «à la jouissance des particuliers d'empêchement que bien à propos » (p.618). Nous voyons ici à l'œuvre le cadre conceptuel dans lequel Vauban avance ses propositions et projets: dépasser les contradictions inhérentes à toute pratique par une juste pondération des avantages et des contraintes qui ne s'obtient qu'en réduisant les données en grandeurs calculables. On a pu se demander si une passion ludique pour le calcul ne l'emporte pas parfois au-delà des possibilités d'anticiper rationnellement. À vrai dire, il reste bien en deçà de l'enthousiasme computationnel de certains de ses contemporains, "statisticiens » avant la lettre. Il est nonobstant bien de ceux qui fournirent alors les premiers exemples de la « statistique », science d'État et science des dénombrements, inventaires et recensements qui décrivent et anticipent en produisant des grandeurs et proportions mathématiques. Il le fait, nous l'avons vu, pour la gestion des forêts, aussi dans sa "Description géographique de la province du Vézelay » (tome II), dans sa fameuse "Cochonnerie ou calcul estimatif pour connaître jusqu'où peut aller la production d'une truie pendant dix années de temps " (tome IV) ou, selon des visées de peuplement, dans ses «Moyens de rétablir nos colonies d'Amérique » (tome IV). En tout état de cause, le projet qui porte ces calculs jusqu'à la plus vaste ambition est celui du perfectionnement du prélèvement fiscal que Vauban engage avec son projet de capitation de 1695 (tome II), jusqu'au projet de Dixme royale, publié en 1707 (tome V). Conçu pour le soulagement du peuple et la gloire du roi, il «réduit les revenus du roi à une proportion géométrique ».

7 La ténacité légendaire de Vauban, mais aussi, à l'évidence, son plaisir à pousser aussi loin que possible ses idées, nous en goûtons tout le sel au fil de ces Mémoires. Chacun nous invite à d'autres réflexions, à d'autres idées, tant est entrainante la force de penser qui y prévaut. Et ceci, même lorsque nous y décelons quelque excès de confiance dans les pouvoirs du calcul, ou encore la marque des préjugés de sa position dans la société de son temps. Et c'est peut-être bien cette adéquation particulière au monde dans lequel il vit qui confère à sa pensée une cohérence telle qu'elle peut devenir l'occasion, pour le lecteur d'aujourd'hui, de réflexions qui nous sont actuelles.

8 Vauban est un grand ingénieur qui a profondément marqué l'histoire de la pensée technique. Avec les outils conceptuels dont il disposait, il a su synthétiser le meilleur de ce que lui transmettait la tradition des ingénieurs, depuis les mécaniciens grecs, les ingénieurs de la Renaissance et du XVII ${ }^{\mathrm{e}}$ siècle. Mais il a su y intégrer, outre les nouvelles orientations que proposaient la philosophie et la science mécanistes, avec la confiance renouvelée dans ses résultats, une largeur de vue politique portée tout à la fois par son sens de l'État et la connaissance du pays, dans toutes ses dimensions et ses diversités, religieuses, sociales, géographiques. Vauban n'a cessé d'y réfléchir, pour chacun de ses habitants, les conditions d'un mieux. 


\section{AUTEURS}

\section{HÉLÈNE VÉRIN}

Centre Alexandre Koyré-CNRS 